\title{
Almost sure exponential stability of an explicit stochastic orthogonal Runge-Kutta-Chebyshev method for stochastic delay differential equations
}

Qian Guo* and Juan Zhong

"Correspondence: qguo@shnu.edu.cn Department of Mathematics, Shanghai Normal University, Shanghai, 200234, China

\begin{abstract}
Compared with Euler-Maruyama type schemes, there is a lack of studies on the stability of Runge-Kutta type methods applied to stochastic delay differential equations (SDDEs). This paper is concerned with filling this imbalance. The focus is on the almost sure exponential stability of an explicit stochastic Runge-Kutta-Chebyshev (S-ROCK) method for an Itô-type linear test equation, which is analyzed by applying the techniques based on a discrete semimartingale convergence theorem.

MSC: 65C30; 60H10; 65LO6

Keywords: stochastic delay differential equations; discrete semimartingale convergence theorem; almost sure stability; Chebyshev method; Runge-Kutta method; explicit schemes
\end{abstract}


of the form

$$
\mathrm{d} y=f(y(t), y(t-\tau)) \mathrm{d} t+g(y(t), y(t-\tau)) \mathrm{d} w(t)
$$

for every $t \geq 0$. Here time delay $\tau>0$. The initial function $y(t)=\psi(t)$ when $t \in[-\tau, 0]$. We further assume that the initial data is independent of Wiener measure driving the equation and $w(t)$ is a scalar Brownian motion on the complete probability space $\left(\Omega, \mathcal{F}, \mathcal{F}_{t \geq 0}, \mathbb{P}\right)$ with a filtration satisfying the usual conditions. Moreover, $f, g: \mathbb{R} \times \mathbb{R} \rightarrow \mathbb{R}$ are Borelmeasurable functions.

The rest of this paper is organized as follows. In the next section, we propose the S-ROCK method for SDDEs. Our main stability results will be derived in Section 3.

\section{The S-ROCK method and preliminary results}

In the following, we employ an equidistant step points $\mathcal{I}_{\Delta t}=\left\{t_{0}, t_{1}, \ldots, t_{N}\right\}$ where the time step size is a submultiple of the delay $\tau$, i.e., $\Delta t=\tau / m$ for a given positive integer $m$, and the $n$th step point is denoted by $t_{n}=n \Delta t$ for $0 \leq n \leq N$. The numerical approximation of $y(t)$ at $t_{n}$ is denoted by $Y_{n}$, and we denote the increment $w\left(t_{n+1}\right)-w\left(t_{n}\right)$ by $J_{n}$. Next we introduce the S-ROCK method for solving SDDEs (1.1), which is given by

$$
Y_{n+1}=\frac{2 \Delta t}{\nu^{2}} f\left(K_{n}^{(\nu)}, Z_{n}^{(\nu)}\right)+2 K_{n}^{(\nu)}-K_{n}^{(v-1)}+J_{n}(1-2 \alpha) g\left(K_{n}^{(v-1)}, Z_{n}^{(\nu-1)}\right),
$$

where $\left\{K_{n}^{(i)}\right\}$ and $\left\{Z_{n}^{(i)}\right\}$ are the stage values defined by

$$
\begin{aligned}
& K_{n}^{(1)}=Y_{n}, \\
& K_{n}^{(2)}=Y_{n}+\frac{\Delta t}{v^{2}} f\left(K_{n}^{(1)}, Z_{n}^{(1)}\right), \\
& K_{n}^{(i)}=\frac{2 \Delta t}{\nu^{2}} f\left(K_{n}^{(i-1)}, Z_{n}^{(i-1)}\right)+2 K_{n}^{(i-1)}-K_{n}^{(i-2)} \text { for } i=3, \ldots, v-1, \\
& K_{n}^{(v)}=\frac{2 \Delta t}{\nu^{2}} f\left(K_{n}^{(v-1)}, Z_{n}^{(\nu-1)}\right)+2 K_{n}^{(\nu-1)}-K_{n}^{(\nu-2)}+J_{n} \alpha g\left(K_{n}^{(\nu-1)}, Z_{n}^{(\nu-1)}\right),
\end{aligned}
$$

and

$$
Z_{n}^{(i)}= \begin{cases}\psi\left(t_{n}+\beta_{i} \Delta t-\tau\right), & t_{n}+\beta_{i} \Delta t-\tau \leq 0 \\ K_{n-m}^{(i)}, & t_{n}+\beta_{i} \Delta t-\tau>0\end{cases}
$$

Here parameters $\alpha \in[0,1 / 2]$ and $\beta_{i}=(i-1)^{2} / \nu^{2}$ for $i=1, \ldots, v$.

Let $C([-\tau, 0] ; \mathbb{R})$ be the family of continuous functions $\varphi$ from $[-\tau, 0]$ to $\mathbb{R}$, equipped with the supremum norm $\|\varphi\|=\sup _{-\tau \leq \theta \leq 0}|\varphi(\theta)|$. Also, denote by $C_{\mathcal{F}_{0}}^{b}([-\tau, 0] ; \mathbb{R})$ the family of bounded, $\mathcal{F}_{0}$-measurable, $C([-\tau, 0] ; \mathbb{R})$-valued random variables.

Now we give some definitions on the almost sure exponential stability of exact and numerical solutions to SDDEs ( $c f$. [16]).

Definition 2.1 The solution $y(t, \psi)$ to SDDEs (1.1) is said to be almost surely exponentially stable if there exists a constant $\eta>0$ such that

$$
\limsup _{t \rightarrow \infty} \frac{1}{t} \log |y(t, \psi)| \leq-\eta \quad \text { a.s. }
$$

for any initial data $\psi \in C_{\mathcal{F}_{0}}^{b}([-\tau, 0] ; \mathbb{R})$. 
Definition 2.2 The solution $Y_{n}$ to numerical scheme (2.1) is said to be almost surely exponentially stable if there exists a constant $\gamma>0$ such that

$$
\limsup _{n \rightarrow \infty} \frac{1}{n \Delta t} \log \left|Y_{n}\right| \leq-\gamma \quad \text { a.s. }
$$

for any bounded variables $\psi(\vartheta \Delta t)$ when $\vartheta \Delta t \in[-\tau, 0]$.

For the purpose of stability, we assume that $f(0,0)=g(0,0)=0$, which implies that (1.1) admits the equilibrium solution $y(t)=0$ corresponding to the initial condition $\psi(t)=0$ for $t \in[-\tau, 0]$. As a standing hypothesis, we shall impose the following local Lipschitz condition ( $c f$. [7]) on the coefficients $f$ and $g$.

(A1) For each integer $D$, there exists a positive constant $K_{D}$ such that, for all

$y_{1}, y_{2}, z_{1}, z_{2} \in R$ with $\left|y_{1}\right| \vee\left|y_{2}\right| \vee\left|z_{1}\right| \vee\left|z_{2}\right| \leq D$,

$\left|f\left(y_{1}, z_{1}\right)-f\left(y_{2}, z_{2}\right)\right|^{2} \vee\left|g\left(y_{1}, z_{1}\right)-g\left(y_{2}, z_{2}\right)\right|^{2} \leq K_{D}\left(\left|y_{1}-y_{2}\right|^{2}+\left|z_{1}-z_{2}\right|^{2}\right)$, where $\vee$ is the maximal operator.

In what follows we introduce the result of almost sure stability of SDDEs (1.1). The proof of the following lemma can be found in [4].

Lemma 2.3 Let Assumptions (A1) hold. Assume that there are four nonnegative constants $\lambda_{1}, \ldots, \lambda_{4}$ such that

$$
\begin{aligned}
& 2 y f(y, 0) \leq-\lambda_{1}|y|^{2}, \\
& |f(y, z)-f(y, 0)| \leq \lambda_{2}|z|, \\
& |g(y, z)|^{2} \leq \lambda_{3}|y|^{2}+\lambda_{4}|z|^{2}
\end{aligned}
$$

for $y, z \in \mathbb{R}$. If

$$
\lambda_{1}>2 \lambda_{2}+\lambda_{3}+\lambda_{4}
$$

then the trivial solution of (1.1) is almost surely exponentially stable.

To explain our idea, we cite the discrete semimartingale convergence theorem as follows (see also [4]).

Theorem 2.4 Let $\left\{A_{j}\right\},\left\{U_{j}\right\}$ be two sequences of nonnegative random variables such that both $A_{j}$ and $U_{j}$ are $\mathcal{F}_{j-1}$-measurable for $j=1,2, \ldots$, and $A_{0}=U_{0}=0$ a.s. Let $\mathcal{M}_{j}$ be a realvalue local martingale with $\mathcal{M}_{0}=0$ a.s. Let $\zeta$ be a nonnegative $\mathcal{F}_{0}$-measurable random variable. Assume that $\left\{X_{j}\right\}$ is a nonnegative semimartingale with the Doob-Mayer decomposition

$$
X_{j}=\zeta+A_{j}-U_{j}+\mathcal{M}_{j}
$$

If $\lim _{j \rightarrow+\infty} A_{j}<+\infty$ a.s. then for almost all $\omega \in \Omega$,

$$
\lim _{j \rightarrow+\infty} X_{j}<+\infty \text { and } \lim _{j \rightarrow+\infty} U_{j}<+\infty
$$




\section{Almost sure asymptotic exponential stability of numerical solution}

Consider the linear SDDE

$$
\mathrm{d} y(t)=(a y(t)+d y(t-\tau)) \mathrm{d} t+(b y(t)+c y(t-\tau)) \mathrm{d} w(t) .
$$

It seems that the stability of approximate solutions to (3.1) using Runge-Kutta type methods is still an open problem. Here we consider the almost sure stability of the linear equation

$$
\mathrm{d} y(t)=a y(t) \mathrm{d} t+(b y(t)+c y(t-\tau)) \mathrm{d} w(t)
$$

which is the same test model as [17]. In this section, our aim is to examine how the S-ROCK method can reproduce the almost sure exponential stability of the exact solution of (3.2). By applying Lemma 2.3, the exact solution of (3.2) is almost surely exponentially stable when $a<-\left(b^{2}+c^{2}\right)$. Now we give a main result of the almost sure stability of the approximate solution (2.1).

Theorem 3.1 Suppose that the conditions of Lemma 2.3 are satisfied. Then the approximate solution (2.1) applied to test model (3.2) is almost surely exponentially stable if the step size $\Delta t$ satisfies

$$
\left[T_{v}\left(1+\frac{a \Delta t}{v^{2}}\right)\right]^{2}+2 \Delta t\left(b^{2}+c^{2}\right)\left(1+2 \alpha \frac{a \Delta t}{v^{2}}\right)^{2}\left[T_{v-2}\left(1+\frac{a \Delta t}{v^{2}}\right)\right]^{2}<1,
$$

where $T_{v}(x)$ is defined as a Chebyshev polynomial of the first kind of degree $v$.

Proof Applying (2.2) to test model (3.2), we have $K_{n}^{(1)}=T_{0}\left(1+\frac{a \Delta t}{v^{2}}\right) Y_{n}$ and $K_{n}^{(2)}=T_{1}(1+$ $\left.\frac{a \Delta t}{v^{2}}\right) Y_{n}$. Next, by the three-term recurrence relation for Chebyshev polynomials, it is easy to prove that

$$
K_{n}^{(i)}=T_{i-1}\left(1+\frac{a \Delta t}{v^{2}}\right) Y_{n}
$$

for $i=1, \ldots, v-1$. Then, from (2.2), we have

$$
\begin{aligned}
K_{n}^{(v)}= & 2 \frac{\Delta t}{v^{2}} a K_{n}^{(v-1)}+2 K_{n}^{(v-1)}-K_{n}^{(v-2)}+J_{n} \alpha\left[b K_{n}^{(v-1)}+c Z_{n}^{(v-1)}\right] \\
= & 2\left(1+\frac{a \Delta t}{v^{2}}\right) T_{\nu-2}\left(1+\frac{a \Delta t}{v^{2}}\right) Y_{n}-T_{\nu-3}\left(1+\frac{a \Delta t}{v^{2}}\right) Y_{n}+J_{n} \alpha b T_{\nu-2}\left(1+\frac{a \Delta t}{v^{2}}\right) Y_{n} \\
& +J_{n} \alpha c Z_{n}^{(v-1)} \\
= & T_{\nu-1}\left(1+\frac{a \Delta t}{v^{2}}\right) Y_{n}+J_{n} \alpha\left[b T_{\nu-2}\left(1+\frac{a \Delta t}{v^{2}}\right) Y_{n}+c Z_{n}^{(v-1)}\right] .
\end{aligned}
$$

Similarly, we have

$$
\begin{aligned}
Y_{n+1} & =2\left(1+\frac{a \Delta t}{v^{2}}\right) K_{n}^{(\nu)}-K_{n}^{(v-1)}+J_{n}(1-2 \alpha)\left[b K_{n}^{(\nu-1)}+c Z_{n}^{(\nu-1)}\right] \\
& =T_{\nu}\left(1+\frac{a \Delta t}{v^{2}}\right) Y_{n}+J_{n}\left(1+2 \frac{a \Delta t}{v^{2}} \alpha\right)\left[b T_{\nu-2}\left(1+\frac{a \Delta t}{v^{2}}\right) Y_{n}+c Z_{n}^{(\nu-1)}\right] .
\end{aligned}
$$


Note that

$$
\begin{aligned}
\left|Y_{n+1}\right|^{2}= & \left|Y_{n}\right|^{2}+\left\{\left[T_{v}\left(1+\frac{a \Delta t}{v^{2}}\right)\right]^{2}-1\right\}\left|Y_{n}\right|^{2} \\
& +\left(J_{n}^{2}-\Delta t\right)\left(1+2 \frac{a \Delta t}{v^{2}} \alpha\right)^{2}\left[b T_{v-2}\left(1+\frac{a \Delta t}{v^{2}}\right) Y_{n}+c Z_{n}^{(v-1)}\right]^{2} \\
& +\Delta t\left(1+2 \frac{a \Delta t}{v^{2}} \alpha\right)^{2}\left[b T_{v-2}\left(1+\frac{a \Delta t}{v^{2}}\right) Y_{n}+c Z_{n}^{(v-1)}\right]^{2} \\
& +2 T_{v}\left(1+\frac{a \Delta t}{v^{2}}\right)\left(1+2 \frac{a \Delta t}{v^{2}} \alpha\right)\left[b T_{v-2}\left(1+\frac{a \Delta t}{v^{2}}\right) Y_{n}+c Z_{n}^{(v-1)}\right] Y_{n} J_{n} \\
\leq & \left|Y_{n}\right|^{2}+\left\{\left[T_{v}\left(1+\frac{a \Delta t}{v^{2}}\right)\right]^{2}-1\right\}\left|Y_{n}\right|^{2}+M_{n} \\
& +2 \Delta t\left(1+2 \frac{a \Delta t}{v^{2}} \alpha\right)^{2}\left\{\left[b T_{v-2}\left(1+\frac{a \Delta t}{v^{2}}\right) Y_{n}\right]^{2}+\left[c Z_{n}^{(v-1)}\right]^{2}\right\},
\end{aligned}
$$

where $M_{n}=\left(J_{n}^{2}-\Delta t\right)\left(1+2 \frac{a \Delta t}{v^{2}} \alpha\right)^{2}\left[b T_{\nu-2}\left(1+\frac{a \Delta t}{v^{2}}\right) Y_{n}+c Z_{n}^{(v-1)}\right]^{2}+2 T_{v}\left(1+\frac{a \Delta t}{v^{2}}\right)\left(1+2 \frac{a \Delta t}{v^{2}} \alpha\right) \times$ $\left[b T_{v-2}\left(1+\frac{a \Delta t}{v^{2}}\right) Y_{n}+c Z_{n}^{(v-1)}\right] Y_{n} J_{n}$.

For any positive constant $C>1$, we have

$$
C^{(\ell+1) \Delta t}\left|Y_{\ell+1}\right|^{2}-C^{\ell \Delta t}\left|Y_{\ell}\right|^{2}=C^{(\ell+1) \Delta t}\left(\left|Y_{\ell+1}\right|^{2}-\left|Y_{\ell}\right|^{2}\right)+\left(C^{(\ell+1) \Delta t}-C^{\ell \Delta t}\right)\left|Y_{\ell}\right|^{2}
$$

Therefore, by (3.5) and (3.6), we obtain

$$
\begin{aligned}
C^{(\ell+1) \Delta t}\left|Y_{\ell+1}\right|^{2}-C^{\ell \Delta t}\left|Y_{\ell}\right|^{2} \\
\leq C^{(\ell+1) \Delta t}\left\{-C^{-\Delta t}+\left[T_{\nu}\left(1+\frac{a \Delta t}{v^{2}}\right)\right]^{2}\right\}\left|Y_{\ell}\right|^{2}+C^{(\ell+1) \Delta t} M_{\ell} \\
\quad+2 C^{(\ell+1) \Delta t} b^{2} \Delta t\left(1+2 \frac{a \Delta t}{v^{2}} \alpha\right)^{2}\left[T_{v-2}\left(1+\frac{a \Delta t}{v^{2}}\right)\right]^{2}\left|Y_{\ell}\right|^{2} \\
\quad+2 C^{(\ell+1) \Delta t} c^{2} \Delta t\left(1+2 \frac{a \Delta t}{v^{2}} \alpha\right)^{2}\left|Z_{\ell}^{(v-1)}\right|^{2}
\end{aligned}
$$

for any nonnegative integer $\ell$. Summing up both sides of inequality (3.7) from $\ell=0$ to $n-1(n \geq 1)$, we have

$$
\begin{aligned}
C^{n \Delta t}\left|Y_{n}\right|^{2} \leq & \left|Y_{0}\right|^{2}+\sum_{\ell=0}^{n-1} C^{(\ell+1) \Delta t} M_{\ell}+\left\{-C^{-\Delta t}+\left[T_{v}\left(1+\frac{a \Delta t}{v^{2}}\right)\right]^{2}\right. \\
& \left.+2 b^{2} \Delta t\left(1+2 \frac{a \Delta t}{v^{2}} \alpha\right)^{2}\left[T_{\nu-2}\left(1+\frac{a \Delta t}{v^{2}}\right)\right]^{2}\right\} \sum_{\ell=0}^{n-1} C^{(\ell+1) \Delta t}\left|Y_{\ell}\right|^{2} \\
& +2 c^{2} \Delta t\left(1+2 \frac{a \Delta t}{v^{2}} \alpha\right)^{2} \sum_{\ell=0}^{n-1} C^{(\ell+1) \Delta t}\left|Z_{\ell}^{(v-1)}\right|^{2} .
\end{aligned}
$$


Let $\mathcal{M}_{n}=\sum_{\ell=0}^{n-1} C^{(\ell+1) \Delta t} M_{\ell}$. Note that the expectation values $E\left(J_{n-1}^{2}-\Delta t\right)=0, E\left(J_{n-1}\right)=0$, moreover, $Y_{n-1}$ and $Z_{n-1}^{(v-1)}$ are $\mathcal{F}_{(n-1) \Delta t}$-measurable, then we have

$$
\begin{aligned}
E\left[\mathcal{M}_{n} \mid \mathcal{F}_{(n-1) \Delta t}\right] & =\mathcal{M}_{n-1}+C^{n \Delta t} E\left[M_{n-1} \mid \mathcal{F}_{(n-1) \Delta t}\right] \\
& =\mathcal{M}_{n-1},
\end{aligned}
$$

which implies that $\mathcal{M}_{n}$ is a martingale with $\mathcal{M}_{0}=0$.

When $t_{n} \leq \tau$, from (2.3) and (3.8), we have

$$
\begin{aligned}
C^{n \Delta t}\left|Y_{n}\right|^{2} \leq & \left|Y_{0}\right|^{2}+\mathcal{M}_{n}+\left\{-C^{-\Delta t}+\left[T_{\nu}\left(1+\frac{a \Delta t}{v^{2}}\right)\right]^{2}\right. \\
& \left.+2 b^{2} \Delta t\left(1+2 \frac{a \Delta t}{v^{2}} \alpha\right)^{2}\left[T_{\nu-2}\left(1+\frac{a \Delta t}{v^{2}}\right)\right]^{2}\right\} \sum_{\ell=0}^{n-1} C^{(\ell+1) \Delta t}\left|Y_{\ell}\right|^{2} \\
& +2 c^{2} \Delta t\left(1+2 \frac{a \Delta t}{v^{2}} \alpha\right)^{2} \sum_{\ell=0}^{n-1} C^{(\ell+1) \Delta t} \psi^{2}\left(t_{\ell}+\beta_{\nu-1} \Delta t-\tau\right) .
\end{aligned}
$$

According to Theorem 2.4, we denote the right side of inequality (3.9) by $X_{n}$. Then, let $\zeta=\left|Y_{0}\right|^{2}+2 c^{2} \Delta t\left(1+2 \frac{a \Delta t}{v^{2}} \alpha\right)^{2} \sum_{\ell=0}^{n-1} C^{(\ell+1) \Delta t} \psi^{2}\left(t_{\ell}+\beta_{\nu-1} \Delta t-\tau\right), U_{n}=0$, and

$$
A_{n}=H_{1}(C) \sum_{\ell=0}^{n-1} C^{(\ell+1) \Delta t}\left|Y_{\ell}\right|^{2}
$$

where $H_{1}(C)=-C^{-\Delta t}+\left[T_{v}\left(1+\frac{a \Delta t}{v^{2}}\right)\right]^{2}+2 b^{2} \Delta t\left(1+2 \frac{a \Delta t}{v^{2}} \alpha\right)^{2}\left[T_{v-2}\left(1+\frac{a \Delta t}{v^{2}}\right)\right]^{2}$. There exists a unique $C^{*}>1$ such that $H_{1}\left(C^{*}\right)=0$ if

$$
\left[T_{v}\left(1+\frac{a \Delta t}{v^{2}}\right)\right]^{2}+2 b^{2} \Delta t\left(1+2 \frac{a \Delta t}{v^{2}} \alpha\right)^{2}\left[T_{v-2}\left(1+\frac{a \Delta t}{v^{2}}\right)\right]^{2}<1
$$

Applying Theorem 2.4, we therefore have $\lim _{n \rightarrow \infty} X_{n}<+\infty$, which means

$$
\lim _{n \rightarrow \infty}\left(C^{*}\right)^{n \Delta t}\left|Y_{n}\right|^{2}<+\infty
$$

When $t_{n}>\tau$, that is, $n>m$, we have

$$
\begin{aligned}
\sum_{\ell=0}^{n-1} C^{(\ell+1) \Delta t}\left|Z_{\ell}^{(\nu-1)}\right|^{2}= & \sum_{\ell=0}^{m-1} C^{(\ell+1) \Delta t} \psi^{2}\left(t_{\ell}+\beta_{\nu-1} \Delta t-\tau\right) \\
& +\sum_{\ell=0}^{n-1} C^{(\ell+m+1) \Delta t}\left|K_{\ell}^{(\nu-1)}\right|^{2}-\sum_{\ell=n-m}^{n-1} C^{(\ell+m+1) \Delta t}\left|K_{\ell}^{(\nu-1)}\right|^{2} .
\end{aligned}
$$

Then, by using (3.4) and (3.8), we have

$$
C^{n \Delta t}\left|Y_{n}\right|^{2}+2 c^{2} \Delta t\left(1+2 \frac{a \Delta t}{v^{2}} \alpha\right)^{2} \sum_{\ell=n-m}^{n-1} C^{(\ell+m+1) \Delta t}\left|K_{\ell}^{(\nu-1)}\right|^{2} \leq X_{n}
$$


and

$$
X_{n}=\zeta_{0}+\mathcal{M}_{n}+H_{2}(C) \sum_{\ell=0}^{n-1} C^{(\ell+1) \Delta t}\left|Y_{\ell}\right|^{2},
$$

where $\zeta_{0}=\left|Y_{0}\right|^{2}+2 c^{2} \Delta t\left(1+2 \frac{a \Delta t}{v^{2}} \alpha\right)^{2} \sum_{\ell=0}^{m-1} C^{(\ell+1) \Delta t} \psi^{2}\left(t_{\ell}+\beta_{v-1} \Delta t-\tau\right), H_{2}(C)=H_{1}(C)+$ $2 c^{2} \Delta t\left(1+2 \frac{a \Delta t}{v^{2}} \alpha\right)^{2} C^{m \Delta t}\left[T_{\nu-2}\left(1+\frac{a \Delta t}{v^{2}}\right)\right]^{2}$.

Note that

$$
\begin{aligned}
& H_{2}(1)=-1+\left[T_{v}\left(1+\frac{a \Delta t}{v^{2}}\right)\right]^{2}+2 \Delta t\left(b^{2}+c^{2}\right)\left(1+2 \frac{a \Delta t}{v^{2}} \alpha\right)^{2}\left[T_{\nu-2}\left(1+\frac{a \Delta t}{v^{2}}\right)\right]^{2}, \\
& H_{2}^{\prime}(C)=\Delta t C^{(-\Delta t-1)}+2 c^{2} m(\Delta t)^{2}\left(1+2 \frac{a \Delta t}{v^{2}} \alpha\right)^{2}\left[T_{\nu-2}\left(1+\frac{a \Delta t}{v^{2}}\right)\right]^{2} C^{(m \Delta t-1)}>0
\end{aligned}
$$

for any $C>1$, and $H_{2}(\infty)>0$.

Obviously, the condition (3.3) yields $H_{2}(1)<0$, which implies that there exists a unique $C^{*}>1$ such that $H_{2}\left(C^{*}\right)=0$. We therefore have $\lim _{n \rightarrow \infty} X_{n}<+\infty$ with Theorem 2.4, which means

$$
\lim _{n \rightarrow \infty}\left(C^{*}\right)^{n \Delta t}\left|Y_{n}\right|^{2} \leq \lim _{n \rightarrow \infty} X_{n}<+\infty
$$

by (3.12). Choose the $\gamma>0$, such that $C^{*}=e^{\gamma}$ and hence

$$
\lim _{n \rightarrow \infty} e^{\gamma n \Delta t}\left|Y_{n}\right|^{2}<+\infty
$$

We therefore obtain

$$
\lim _{n \rightarrow \infty} \frac{1}{n \Delta t} \log \left|Y_{n}\right| \leq-\frac{\gamma}{2}, \quad \text { a.s. }
$$

as required.

Finally, (3.3) also implies (3.10). This completes the proof of Theorem 3.1.

Next, we state how to choose a parameter $\alpha$ and the stage number $v$ to obtain almost surely stable numerical solution based on Theorem 3.1.

Corollary 3.2 Suppose that conditions of Lemma 2.3 are satisfied. The approximate solution (2.1) applied to test model (3.2) is almost surely exponentially stable if we choose the parameter $\alpha=-\frac{v^{2}}{2 a \Delta t}$ and a $\Delta$ t satisfies

$$
-2 v^{2}<a \Delta t \leq-v^{2} \quad \text { and } \quad T_{v}\left(1+\frac{a \Delta t}{v^{2}}\right) \neq \pm 1
$$

where stage number $v \geq 2$.

Proof The inequality $a \Delta t \leq-v^{2}$ guarantees that $-\frac{v^{2}}{2 a \Delta t} \in(0,1 / 2]$, hence choosing $\alpha=$ $-\frac{v^{2}}{2 a \Delta t}$ satisfies the definition of the S-ROCK method and also simplifies the left hand side of (3.3) into $\left[T_{v}\left(1+\frac{a \Delta t}{v^{2}}\right)\right]^{2}$. Finally, $\left[T_{v}\left(1+\frac{a \Delta t}{v^{2}}\right)\right]^{2}<1$ if $-2 v^{2}<a \Delta t<0$ and $T_{v}\left(1+\frac{a \Delta t}{v^{2}}\right) \neq \pm 1$ 
such that the inequality (3.3) is valid. This completes the proof of Corollary 3.2 by using Theorem 3.1.

Now we consider the case $-4<a \Delta t<0$.

To guarantee the sufficient condition (3.3), $\left(1+2 \frac{a \Delta t}{v^{2}} \alpha\right)^{2}$ should be as small as possible such that (3.3) is valid. Therefore, it is a good choice to set $\alpha=1 / 2$ and a small $v$ because $\left(1+2 \frac{a \Delta t}{v^{2}} \alpha\right)^{2}$ is a monotonically decreasing and continuous function of the parameter $\alpha$ on $[0,1 / 2]$.

\section{Competing interests}

The authors declare that they have no competing interests.

\section{Authors' contributions}

The main idea of this paper was proposed by QG and QG wrote the paper. JZ participated in the proof of Theorem 3.1 and helped to draft the manuscript. All authors read and approved the final manuscript.

\section{Acknowledgements}

This work was partially supported by E-Institutes of Shanghai Municipal Education Commission (No. E03004), Natural Science Foundation of Shanghai (No. 14ZR1431300) and Innovation Program of Shanghai Municipal Education Commission (No. 14YZ078).

Received: 22 May 2015 Accepted: 22 September 2015 Published online: 30 September 2015

\section{References}

1. Rodkina, A, Schurz, H: Almost sure asymptotic stability of drift-implicit $\theta$-methods for bilinear ordinary stochastic differential equation in $\mathbb{R}^{1}$. J. Comput. Appl. Math. 180, 13-31 (2005)

2. Higham, DJ, Mao, X, Yuan, C: Almost sure and moment exponential stability in the numerical simulation of stochastic differential equation. SIAM J. Numer. Anal. 45, 592-609 (2007)

3. Pang, S, Deng, F, Mao, X: Almost sure and moment exponential stability of Euler-Maruyama discretizations for hybrid stochastic differential equations. J. Comput. Appl. Math. 213, 127-141 (2008)

4. Wu, F, Mao, X, Szpruch, L: Almost sure exponential stability of numerical solutions for stochastic delay differential equations. Numer. Math. 115, 681-697 (2010)

5. Wu, F, Mao, X, Kloeden, PE: Almost sure exponential stability of the Euler-Maruyama approximations for stochastic functional differential equations. Random Oper. Stoch. Equ. 19, 165-186 (2011)

6. Schurz, H: Almost sure asymptotic stability and convergence of stochastic theta methods applied to system of linear SDEs in $\mathbb{R}^{d}$. Random Oper. Stoch. Equ. 19, 111-129 (2011)

7. Mao, X, Szpruch, L: Strong convergence and stability of implicit numerical methods for stochastic differential equations with non-globally Lipschitz continuous coefficients. J. Comput. Appl. Math. 238, 14-28 (2013)

8. Saito, Y, Mitsui, T: Stability analysis of numerical schemes for stochastic differential equations. SIAM J. Numer. Anal. 33 2254-2267 (1996)

9. Burrage, K, Burrage, P, Mitsui, T: Numerical solutions of stochastic differential equations implementation and stability issues. J. Comput. Appl. Math. 125, 171-182 (2000)

10. Cao, WR, Liu, MZ, Fan, ZC: MS-stability of the Euler-Maruyama method for stochastic differential delay equations. Appl. Math. Comput. 159, 127-135 (2004)

11. Baker, CTH, Buckwar, E: Exponential stability in $p$-th mean of solutions, and of convergent Euler-type solutions, of stochastic delay differential equations. J. Comput. Appl. Math. 184, 404-427 (2005)

12. Mao, X: Exponential stability of equidistant Euler-Maruyama approximations of stochastic differential delay equations. J. Comput. Appl. Math. 200, 297-316 (2007)

13. Huang, CM: Mean square stability and dissipativity of two classes of theta methods for systems of stochastic delay differential equations. J. Comput. Appl. Math. 259, 77-86 (2014)

14. Abdulle, A, Cirilli, S: S-ROCK: Chebyshev methods for stiff stochastic differential equations. SIAM J. Sci. Comput. 30 997-1014 (2008)

15. Abdulle, A, Li, T: S-ROCK methods for stiff Itô SDEs. Commun. Math. Sci. 6, 845-868 (2008)

16. Mao, X: Stochastic Differential Equations and Their Applications. Ellis Horwood, Chichester (1997)

17. Huang, CM, Gan, SQ, Wang, DS: Delay-dependent stability analysis of numerical methods for stochastic delay differential equations. J. Comput. Appl. Math. 236, 3514-3527 (2012) 